\title{
EDITORIAL
}

\section{Image in Headache Medicine: "a picture is worth a thousand words"}

\footnotetext{
Tre

Lhe reader may have noticed that we are publishing a different picture on the front page of each of the issues of 'Headache Medicine'. "A picture is worth a thousand words" is an adage indicating the notion that by simply seeing a picture we can understand a complex idea, or in our case, the pathophysiology of a given disease such as trigeminal neuralgia in the course of multiple sclerosis. It seems that the modern use of the phrase was initiated by Fred R. Barnard in an advertising trade journal (Printers' Ink) using images in advertisements that appeared on the sides of streetcars: "One look is worth a thousand words" (December 8, 1921).

In addition, in the present issue the readers will find interesting articles dealing with cluster headache current understanding and treatment, magnesium ion serum profile in chronic migraine, cognition in women with migraine, migraine in the menopause and perimenopause, quality of life for migraineurs, as well as the correlation between forward head posture and primary headaches in adolescents.
}

\section{Reference}

http://en.wikipedia.org/wiki/A_picture_is_worth_a_thousand_words

Marcelo M. Valença \& Fernando Kowacs 